\title{
Bacteriological profile and antibiogram of blood culture isolates done by automated culture and sensitivity method in a neonatal intensive care unit in a tertiary care hospital in Odisha, India
}

\author{
Kausik Kumar Sarangi ${ }^{1}$, Dipti Pattnaik ${ }^{1}$, Surya Narayan Mishra ${ }^{1}$, \\ Manas Kumar Nayak ${ }^{2}$, Jagadananda Jena ${ }^{1}$
}

\footnotetext{
${ }^{1}$ Department of Microbiology, Kalinga Institute of Medical Sciences, Bhubaneswar, Odisha, India

${ }^{2}$ Department of Paediatrics, Kalinga Institute of Medical Sciences, Bhubaneswar, Odisha, India
}

Received: 15 September 2015

Accepted: 05 October 2015

\section{*Correspondence:}

Dr. Surya Narayan Mishra,

E-mail: surya.mishra@kims.ac.in

Copyright: (C) the author(s), publisher and licensee Medip Academy. This is an open-access article distributed under the terms of the Creative Commons Attribution Non-Commercial License, which permits unrestricted non-commercial use, distribution, and reproduction in any medium, provided the original work is properly cited.

\begin{abstract}
Background: Sepsis is one of the commonest cause of neonatal mortality. The aetiology of neonatal sepsis has variations according to the various customs and practices in the perinatal and neonatal period and geographical area. This study was designed to analyse the magnitude and aetiological characteristics of neonatal sepsis $\&$ also the antibiotic sensitivity pattern of the isolates.

Methods: This prospective study was conducted in the Department of Microbiology including 250 neonates with suspected neonatal sepsis admitted to Dept. of Paediatrics \& NICU, of Kalinga Institute of Medical Sciences (KIMS), Bhubaneswar, during the period from Nov. 2012 - April 2014. Two ml of venous blood was taken from each neonate \& cultured by automated BacT/Alert \& VITEK2 method for rapid isolation \& sensitivity test. A structured proforma was used to collect the information for the baseline characteristics like age, gender, birth weight, gestational age, mode of delivery of the neonate and age of onset of illness.

Results: Amongst the total samples processed, 82(32.8\%) became culture positive among which gram positive bacteria were the most predominant $(65.8 \%)$. Out of all the gram positive pathogens, CoNS were the most common isolates $(88.5 \%)$. Among the total culture positive pathogenic isolates $(\mathrm{n}=82)$, S. haemolyticus was the predominant bacteria 28 (34.1\%) followed by S. epidermidis 12 (14.6\%) and Esch coli. 8 (9.8\%). Staph. aureus was isolated from $6(7.3 \%)$ cases whereas candida spp. were isolated from only 8 cases $(9.6 \%)$.

Conclusions: Present study revealed that both gram positive and gram negative bacteria were responsible for blood stream infections and most of the strains were multidrug resistant. Among gram positive bacterial pathogens, most common were Coagulase Negative Staphylococcus (CoNS) (88.5\%) followed by Staphylococcus aureus $(7.3 \%)$.
\end{abstract}

Keywords: Automated culture, Neonatal sepsis, CoNS

\section{INTRODUCTION}

Neonatal septicaemia refers to a clinical syndrome characterised by systemic signs and symptoms due to generalised bacterial infections with a positive blood culture in the first four weeks of life. ${ }^{1}$ Bacterial infections are the commonest cause of morbidity and mortality during the neonatal period. Fulminate and fatal course of infection may result from complications such as shock, disseminated intravascular coagulation (DIC) and multisystem organ failure, mandating early diagnosis of 
this life threatening condition for a timely treatment and a favourable outcome. ${ }^{2}$

\section{Aims and objectives}

To find out the bacteriological profile and antibiogram of blood culture isolates from neonatal septicaemic cases done by automated culture \& sensitivity method in a Neonatal Intensive Care Unit (NICU) of Kalinga Institute of Medical Sciences (KIMS), Bhubaneswar, Odisha, India.

\section{METHODS}

A prospective study covering 250 suspected neonatal septicaemic cases was carried out in the Department of Microbiology in association with Dept. of Paediatrics \& NICU, of Kalinga Institute of Medical Sciences, Bhubaneswar, during the period from Nov. 2012 - April 2014.

Two $\mathrm{ml}$ of venous blood samples for culture were collected following standard aseptic precautions in specific automated aerobic blood culture bottle [FAN (Fastidious Antibiotic Neutralization bottle (yellow cap) BioMerieux \#259794]. If empirical antibiotics were already started, the collection was timed before the next dose of antibiotic was due or about half an hour before the predicted peak of temperature.

The samples thus collected were transferred immediately to the Microbiology section of Central Laboratory, KIMS and were cultured by automated BacT/Alert \& VITEK2 method for rapid isolation \& sensitivity test. The results thus obtained were analysed to find the significance of various parameters.

The blood culture bottles positive for bacterial growth changed its colour from blue green to yellow \& positive bottles are determined by one of three software algorithms and are indicated as a yellow sensor and high reflectance units. Negative bottles result from darker sensors and low reflectance units.

\section{RESULTS}

Out of 250 clinically suspected septicaemic cases of neonates, $154(61.6 \%)$ were males and 96 (38.4\%) were females. Among the males culture positivity was 45 $(54.9 \%) \&$ for females it was $37(45.1 \%)$. Neonates of the age group 8-28 days showed maximum culture positivity $38(46.3 \%)$. Total culture positivity in the study in all the age group was $82(32.8 \%)$ (Table 1$)$.

Table 1: Association between physical parameters and sepsis.

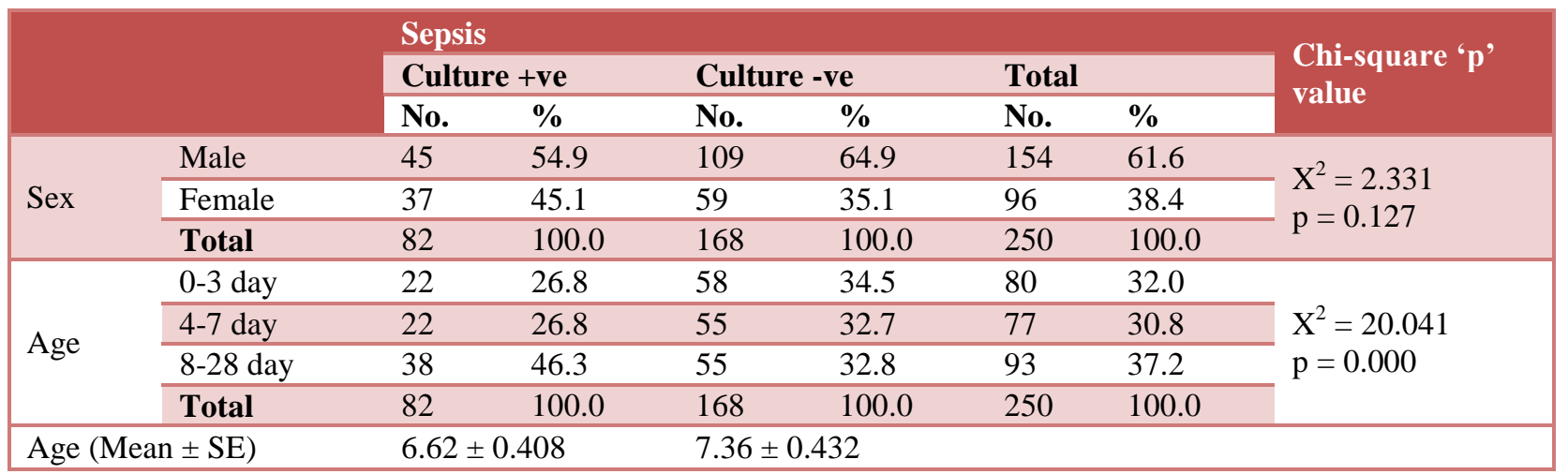

Out of all the culture positive septicaemic cases, maximum percentage of positivity were seen in VLBW group. (60\%) followed by LBW neonates (47.6\%). Maximum bacterial growth was seen in pre term babies (56\%) as compared to the term babies (19.5\%). PROM was also found to be the important risk factor for culture positivity (47.8\%) than the absence of PROM (31.2\%). Home delivery was seen as an important risk factor for sepsis $(46.6 \%)$ than the hospital delivery both at KIMS or in other institutions $(29.5 \% \& 33.8 \%$ respectively). History of caesarean section was seen in $34.9 \%$ of culture positive septicaemic cases as compared to the normal delivery $(28.2 \%)$. Significant $\mathrm{p}$ value was observed in relation to birth weight, gestational age and PROM.
Out of total 82 pathogenic isolates recorded by automated system, S. hemolyticus was the predominant bacteria 28 (34.1\%) followed by S. epidermidis 12 (14.6\%) and Esch. coli. 8 (9.8\%). S. aureus was isolated from $6(7.3 \%)$ cases whereas Candida albicans was also isolated from 8 $(9.8 \%)$ cases (Table 3).

Isolation of Gram positive bacteria $(75.0 \%)$ and fungi $(10 \%)$ were more from late onset septicaemic cases as compared to the early onset septicaemia (31.8\% and $9.0 \%$ ) but Gram negative bacterial pathogens $(59.0 \%)$ were predominant from early onset septicaemic cases (Table 4). 
Table 2: Association of clinically suspected cases by risk factor.

\begin{tabular}{|c|c|c|c|c|c|c|c|c|}
\hline & & \multicolumn{6}{|c|}{ Sepsis } & \multirow{3}{*}{$\begin{array}{l}\text { Chi-square } \\
\text { 'p' value }\end{array}$} \\
\hline & & \multicolumn{2}{|c|}{ Culture +ve } & \multicolumn{2}{|c|}{ Culture -ve } & \multicolumn{2}{|c|}{ Total } & \\
\hline & & No. & $\%$ & No. & $\%$ & No. & $\%$ & \\
\hline \multirow{4}{*}{ Birth weight } & Very LBW & 3 & 60.00 & 2 & 40.00 & 5 & 2.00 & \multirow{4}{*}{$\begin{array}{l}X^{2}=16.811 \\
p=0.000\end{array}$} \\
\hline & LBW & 41 & 48.20 & 44 & 51.80 & 85 & 34.00 & \\
\hline & Normal & 38 & 23.80 & 122 & 76.20 & 160 & 64.00 & \\
\hline & Total & 82 & 32.80 & 168 & 67.20 & 250 & 100.00 & \\
\hline \multirow{3}{*}{ Gestation } & Term & 31 & 19.50 & 128 & 80.50 & 159 & 63.60 & \multirow{3}{*}{$\begin{array}{l}X^{2}=35.072 \\
p=0.000\end{array}$} \\
\hline & Preterm & 51 & 56.00 & 40 & 44.00 & 91 & 36.40 & \\
\hline & Total & 82 & 32.80 & 168 & 67.20 & 250 & 100.00 & \\
\hline \multirow{3}{*}{ PROM } & Absent & 71 & 31.30 & 156 & 68.70 & 227 & 90.80 & \multirow{3}{*}{$\begin{array}{l}X^{2}=2.595 \\
p=0.107\end{array}$} \\
\hline & Present & 11 & 47.80 & 12 & 52.20 & 23 & 9.20 & \\
\hline & Total & 82 & 32.80 & 168 & 67.20 & 250 & 100.00 & \\
\hline \multirow{4}{*}{$\begin{array}{l}\text { Place of } \\
\text { delivery }\end{array}$} & Other Institution & 44 & 33.80 & 86 & 66.20 & 130 & 52.00 & \multirow{4}{*}{$\begin{array}{l}X^{2}=1.884 \\
p=0.039\end{array}$} \\
\hline & KIMS & 31 & 29.50 & 74 & 70.50 & 105 & 42.00 & \\
\hline & Home delivery & 7 & 46.70 & 8 & 53.30 & 15 & 6.00 & \\
\hline & Total & 82 & 32.80 & 168 & 67.20 & 250 & 100.00 & \\
\hline \multirow{3}{*}{$\begin{array}{l}\text { Mode of } \\
\text { delivery }\end{array}$} & $\mathrm{CS}$ & 60 & 34.90 & 112 & 65.10 & 172 & 68.80 & \multirow{3}{*}{$\begin{array}{l}X^{2}=1.086 \\
p=0.297\end{array}$} \\
\hline & ND & 22 & 28.20 & 56 & 71.80 & 78 & 31.20 & \\
\hline & Total & 82 & 32.80 & 168 & 67.20 & 250 & 100.00 & \\
\hline
\end{tabular}

Table 3: Distribution of pathogenic isolates by automated System $(n=82)$.

\begin{tabular}{|lll|}
\hline Bacterial pathogens & No. & $\%$ \\
\hline S. haemolyticus & 28 & 34.10 \\
\hline S. epidermidis & 12 & 14.60 \\
\hline S. werneri & 3 & 3.70 \\
\hline S. hominis & 3 & 3.70 \\
\hline S. aureus & 6 & 7.30 \\
\hline Enterobacter cloacae & 6 & 7.30 \\
\hline Burkholderia cepacia & 4 & 4.90 \\
\hline Acinetobacter lwoffi & 2 & 2.40 \\
\hline S. paratyphi A & 2 & 2.40 \\
\hline Esch. coli & 8 & 9.80 \\
\hline C. albicans & 8 & 9.80 \\
\hline Total & 82 & 100.00 \\
\hline
\end{tabular}

Table 4: Organism type vs. onset of septicaemia.

\begin{tabular}{|llll|}
\hline \multirow{2}{*}{ Organism type } & \multicolumn{3}{l}{ Onset of septicaemia } \\
\cline { 2 - 4 } & EOS & LOS & Total \\
\cline { 2 - 4 } & No. & No. & No. \\
\hline Gram positive & $7(31.8)$ & $45(75 \%)$ & $52(63.1 \%)$ \\
\hline Gram negative & $13(59 \%)$ & $9(15 \%)$ & $222(6.3 \%)$ \\
\hline Fungi & $2(9 \%)$ & $6(10 \%)$ & $8(9.6 \%)$ \\
\hline Total & $22(\mathrm{n}=22)$ & $60(\mathrm{n}=60)$ & $82(\mathrm{n}=82)$ \\
\hline $\begin{array}{l}\text { Chi-square } \\
\text { 'p' value }\end{array}$ & $\mathrm{X}^{2}=16.411 ; \mathrm{p}=0.000$ & \\
\hline
\end{tabular}

Among all the gram positive isolates, CoNS were the predominant pathogen $46(88.5 \%)$ as compared to the other pathogens $6(11.5 \%)$ in this study (Figure 1).

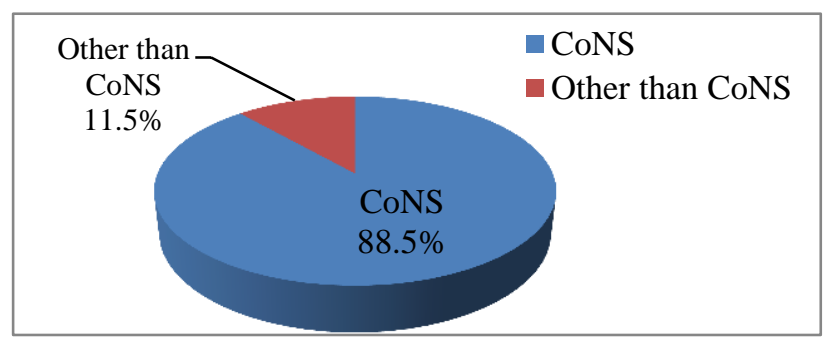

Figure 1: CoNS among Gram positive cases.

All the Gram positive pathogenic isolates were sensitive to linezolid, tigecycline and vancomycin. Co-trimoxazole was sensitive in $78.8 \%$ isolates followed by ceftriaxone (76.9\%), azithromycin (76.9\%), cefepime (65.4\%) erythromycin $(55.8 \%)$ clindamycin $(51.9 \%)$ levofloxacin (42.3\%), amikacin (42.3\%), cefadroxil (30.8\%) and gentamicin (25\%). Maximum resistance was seen against benzylpenicilin (86.6\%) and ampicilin (71.2\%) (Table 5).

Among the Gram negative isolates maximum antibiotic sensitivity was observed for tigecycline (90.9\%), levofloxacin $(86.4 \%)$ \& amikacin $(86.4 \%)$ followed by imipenem $(77.3 \%)$, meropenem $(77.3 \%)$, tobramycin (77.3\%), ciprofloxacin $(68.2 \%)$, piperacilin-tazobactum $(68.2 \%)$, ceftriaxone $(54.5 \%)$ and cefepime $(40.9 \%)$. Resistance was maximum for cefadroxil (86.4\%), ampicilin $(72.7 \%)$ and cefuroxime $(68.2 \%)$ (Table 6). 
Table 5: Antibiotic sensitivity patterns among gram positive isolates (No \& \% showing sensitivity).

\begin{tabular}{|c|c|c|c|c|c|c|c|c|c|c|c|c|}
\hline \multirow{3}{*}{$\begin{array}{l}\text { Name of the } \\
\text { antibiotic }\end{array}$} & \multicolumn{12}{|c|}{ Bacteria isolated in automated system } \\
\hline & \multicolumn{2}{|c|}{$\begin{array}{l}\text { S. haemolyticus } \\
(\mathrm{n}=\mathbf{2 8})\end{array}$} & \multicolumn{2}{|c|}{$\begin{array}{l}\text { S. epidermidis } \\
(\mathrm{n}=12)\end{array}$} & \multicolumn{2}{|c|}{$\begin{array}{l}\text { S. werneri } \\
(\mathrm{n}=3)\end{array}$} & \multicolumn{2}{|c|}{$\begin{array}{l}\text { S. hominis } \\
(\mathrm{n}=\mathbf{3})\end{array}$} & \multicolumn{2}{|c|}{$\begin{array}{l}\begin{array}{l}\text { S. aureus } \\
(\mathbf{n}=\mathbf{6})\end{array}\end{array}$} & \multicolumn{2}{|c|}{$\begin{array}{l}\text { Total } \\
(\mathrm{n}=52)\end{array}$} \\
\hline & No. & $\%$ & No. & $\%$ & No. & $\%$ & No. & $\%$ & No. & $\%$ & No. & $\%$ \\
\hline Linezolid & 28 & 100 & 12 & 100 & 3 & 100 & 3 & 100 & 6 & 100 & 52 & 100 \\
\hline Tigecycline & 28 & 100 & 12 & 100 & 3 & 100 & 3 & 100 & 6 & 100 & 52 & 100 \\
\hline Cefadroxil & 7 & 25 & 5 & 41.7 & 1 & 33.3 & 1 & 33.3 & 2 & 33.3 & 16 & 30.8 \\
\hline Benzyl penicillin & 4 & 14.3 & 2 & 16.7 & 0 & 0 & 0 & 0 & 1 & 16.7 & 7 & 13.5 \\
\hline Clindamycin & 14 & 50 & 7 & 58.3 & 1 & 33.3 & 2 & 66.7 & 3 & 50 & 27 & 51.9 \\
\hline Ciprofloxacin & 9 & 32.1 & 5 & 41.7 & 2 & 66.7 & 1 & 33.3 & 2 & 33.3 & 19 & 36.5 \\
\hline Oxacillin & 14 & 50 & 5 & 41.7 & 2 & 66.7 & 2 & 66.7 & 4 & 66.7 & 27 & 51.9 \\
\hline Gentamicin & 5 & 17.9 & 5 & 41.7 & 1 & 33.3 & 1 & 33.3 & 1 & 16.7 & 13 & 25 \\
\hline Ampicillin & 6 & 21.4 & 3 & 25 & 0 & 0 & 0 & 0 & 3 & 50 & 12 & 23.1 \\
\hline Co-trimoxazole & 22 & 78.6 & 9 & 75 & 3 & 100 & 2 & 66.7 & 5 & 83.3 & 41 & 78.8 \\
\hline Cefuroxime & 10 & 35.7 & 2 & 16.7 & 1 & 33.3 & 2 & 66.7 & 3 & 50 & 18 & 34.6 \\
\hline Ceftriaxone & 20 & 71.4 & 9 & 75 & 3 & 100 & 3 & 100 & 5 & 83.3 & 40 & 76.9 \\
\hline Cefepime & 18 & 64.3 & 8 & 66.7 & 2 & 66.7 & 3 & 100 & 3 & 50 & 34 & 65.4 \\
\hline Amikacin & 10 & 35.7 & 5 & 41.7 & 2 & 66.7 & 2 & 66.7 & 3 & 50 & 22 & 42.3 \\
\hline Azithromicin & 21 & 75 & 9 & 75 & 3 & 100 & 2 & 66.7 & 5 & 83.3 & 40 & 76.9 \\
\hline Erythromycin & 16 & 57.1 & 7 & 58.3 & 2 & 66.7 & 0 & 0 & 4 & 66.7 & 29 & 55.8 \\
\hline Vancomycin & 28 & 100 & 12 & 100 & 3 & 100 & 3 & 100 & 6 & 100 & 52 & 100 \\
\hline
\end{tabular}

Table 6: Antibiotic sensitivity patterns among gram negative isolates (No \& \% showing sensitivity).

\begin{tabular}{|c|c|c|c|c|c|c|c|c|c|c|c|c|}
\hline \multirow{3}{*}{$\begin{array}{l}\text { Name of the } \\
\text { antibiotic }\end{array}$} & \multicolumn{12}{|c|}{ Bacteria isolated in automated system } \\
\hline & \multicolumn{2}{|c|}{$\begin{array}{l}\text { Enterobacter } \\
\text { cloacae }(\mathrm{n}=6)\end{array}$} & \multicolumn{2}{|c|}{$\begin{array}{l}\text { Burkholderia } \\
\text { cepacia }(n=4)\end{array}$} & \multicolumn{2}{|c|}{$\begin{array}{l}\text { Acinetobacter } \\
\text { lwoffi }(\mathrm{n}=2)\end{array}$} & \multicolumn{2}{|c|}{$\begin{array}{l}\text { S. paratyphi A } \\
(\mathrm{n}=2)\end{array}$} & \multicolumn{2}{|c|}{$\begin{array}{l}\text { Esch. coli } \\
(\mathrm{n}=8)\end{array}$} & \multicolumn{2}{|c|}{$\begin{array}{l}\text { Total } \\
(\mathbf{n}=\mathbf{2 2})\end{array}$} \\
\hline & No. & $\%$ & No. & $\%$ & No. & $\%$ & No. & $\%$ & No. & $\%$ & No. & $\%$ \\
\hline Ampicillin & 2 & 33.3 & 0 & 0 & 0 & 0 & 2 & 100 & 2 & 25 & 6 & 27.3 \\
\hline Gentamicin & 2 & 33.3 & 2 & 50 & 1 & 50 & 1 & 50 & 4 & 50 & 10 & 45.5 \\
\hline Amikacin & 5 & 83.3 & 3 & 75 & 2 & 100 & 2 & 100 & 7 & 87.5 & 19 & 86.4 \\
\hline Ciprofloxacin & 5 & 83.3 & 2 & 50 & 1 & 50 & 1 & 50 & 6 & 75 & 15 & 68.2 \\
\hline Levofloxacin & 5 & 83.3 & 3 & 75 & 2 & 100 & 2 & 100 & 7 & 87.5 & 19 & 86.4 \\
\hline Cefadroxil & 1 & 16.7 & 0 & 0 & 0 & 0 & 1 & 50 & 1 & 12.5 & 3 & 13.6 \\
\hline Cefuroxime & 2 & 33.3 & 1 & 25 & 0 & 0 & 2 & 100 & 2 & 25 & 7 & 31.8 \\
\hline Ceftriaxone & 4 & 66.7 & 2 & 50 & 0 & 0 & 2 & 100 & 5 & 62.5 & 13 & 59.1 \\
\hline Cefepime & 3 & 50 & 1 & 25 & 0 & 0 & 2 & 100 & 3 & 37.5 & 9 & 40.9 \\
\hline Tobramicin & 4 & 66.7 & 3 & 75 & 2 & 100 & 2 & 100 & 6 & 75 & 17 & 77.3 \\
\hline $\begin{array}{l}\text { Piperacillin- } \\
\text { Tazobactum }\end{array}$ & 5 & 83.3 & 2 & 50 & 0 & 0 & 2 & 100 & 6 & 75 & 15 & 68.2 \\
\hline Imipenem & 6 & 100 & 1 & 25 & 0 & 0 & 2 & 100 & 8 & 100 & 17 & 77.3 \\
\hline Meropenem & 6 & 100 & 1 & 25 & 0 & 0 & 2 & 100 & 8 & 100 & 17 & 77.3 \\
\hline Tigecycline & 6 & 100 & 2 & 50 & 2 & 100 & 2 & 100 & 8 & 100 & 20 & 90.9 \\
\hline Co-trimoxazole & 5 & 83.3 & 4 & 100 & 1 & 50 & 2 & 100 & 4 & 50 & 16 & 72.7 \\
\hline
\end{tabular}

\section{DISCUSSION}

The present study included 250 clinically suspected neonates suffering from sepsis and admitted to the NICU of Paediatrics Department, KIMS. Distribution of cases based on onset of septicaemia \& type of organism have been analysed. Further, antibiotic sensitivity pattern of the bacterial pathogens have been thoroughly analysed.
In the present study, the total number of LBW babies are 55 out of 250 clinically suspected neonates (21.6\%), amongst which culture positivity was seen in 47 cases $(72.7 .4 \%)$. This finding is nearer to the outcome of the study conducted by Manjushree $\mathrm{H}$ Bhalchandra ${ }^{3}$ where majority of the LBW babies were associated with culture positive sepsis $(60 \%)$. Another study conducted by M. N. 
Shah et al., ${ }^{4}$ showed, the association of LBW neonates \& sepsis as $50 \%$.

The number of VLBW neonates in our study is $5(2 \%)$ out of which culture positivity was $60 \%$ which is closely related to the study conducted by Jia-Horng Jiang et al. where culture positivity of VLBW neonates was $54.4 \%$.

In our study $51(56 \%)$ neonates showed blood culture positivity in association with pre-term delivery. This finding is at par with the study conducted by M. N. Shah et al. ${ }^{4}$ where $51.04 \%$ bacteriologically positive cases were associated with pre-term delivery.

In the present study out of 250 clinically suspected neonates, 82 were bacteriologically confirmed cases among which Staphylococcus haemolyticus was the predominant isolate $28(34.1 \%)$ followed by Staphylococcus epidermidis 12 (14.6\%), S. werneri 3 (3.7\%), S. hominis $3(3.7 \%)$, \& S. aureus $6(7.3 \%)$. Other pathogens isolated were Enterobacter cloacae 6 (7.3\%), Burkholderia cepacia 4 (4.9\%), Acinetobacter lwoffi 2 (2.4\%), Salmonella paratyhi A 2 (2.4\%), Esch coli 8 (9.8\%) \& Candida albicans 8 (9.8\%). It shows that gram positive bacterial pathogens were the commonest isolates $52(63.4 \%)$ followed by Gram negative bacterial pathogens $26.8 \%$ \& fungi $9.8 \%$. This finding is similar to the outcome of the study conducted by Hilal Ozkan et al. ${ }^{6}$ where it was shown that Gram positive isolates were $68.2 \%$ followed by Gram negative isolates (16.5\%) \& fungi (15.8\%).

Regarding onset of sepsis it has been shown in our study that Gram positive bacterial pathogens were more commonly associated with late onset sepsis which accounts to $(72.5 \%)$ \& Gram negative pathogens were associated with early onset sepsis $(59 \%)$ which is close to the findings of the study conducted by F. Motara et al. ${ }^{7}$ where late onset sepsis was most common \& CoNS was the predominant gram positive pathogen $(67.6 \%) \&$ Gram negative isolates as predominant pathogens in early onset sepsis. In another study conducted by Jia-Horng Jiang et al. ${ }^{8}$ CoNS were also associated commonly with late onset sepsis.

In our study all the Gram positive pathogenic isolates were sensitive to linezolid, tigecycline and vancomycin. Co-trimoxazole was sensitive in $78.8 \%$ isolates followed by ceftriaxone $(77 \%)$, azithromycin $(76.9 \%)$, cefepime (60\%), erythromycin $(59.6 \%)$ \& clindamycin $(53.9 \%)$. This finding is closely associated with the study conducted by Shahsanam Gheibi et al. ${ }^{9}$ where maximum sensitivity was found to vancomycin (90\%) \& ciprofloxacin $(78.5 \%)$. Study conducted by Katiyar R et al. ${ }^{10}$ showed maximum resistance to penicillin $(7.41 \%) \&$ ampicillin (18.52\%) which is close to the findings of our study. Similar to our study maximum sensitivity to linezolid $(100 \%)$, vancomycin $(95 \%)$, cefotaxime $(73 \%)$, ceftriaxone $(68 \%) \&$ amikacin $(68 \%)$ was also observed in the study conducted by Maimoona Mustafa et al. ${ }^{11}$
For Gram negative pathogenic isolates, our study showed maximum sensitivity to amikacin $(86.4 \%)$ followed by imipenem $(77.3 \%)$, meropenem $(77.3 \%)$, tobramycin (77.3\%), ciprofloxacin $(68.2 \%)$, piperacilin-tazobactum $(68.2 \%)$, ceftriaxone $(59.1 \%)$ and cefepime $(40.9 \%) \&$ resistance to cefadroxil (86.4\%), ampicilin (72.7\%), cefuroxime $(68.2 \%)$ \& gentamicin $(54.5 \%)$ which was at par with the study conducted by Maimoona Mustafa et al. In another study conducted by A. K. Mane et al. ${ }^{12}$ maximum sensitivity was also found to imipenem (100\%), ciprofloxacin $(66.6 \%)$ \& levofloxacin $(66.6 \%)$. $\&$ maximum resistance was found against ampicillin $(81.5 \%)$ \& gentamicin $(85.2 \%)$.

\section{ACKNOWLEDGEMENTS}

The authors are very much thankful to all the staffs of Department of Microbiology and Paediatrics as well as to the management of Kalinga Institute of Medical Sciences, Bhubaneswar, Odisha, India for their constant support and encouragement in completing the project.

\section{Funding: No funding sources}

Conflict of interest: None declared

Ethical approval: The study was approved by the institutional ethics committee

\section{REFERENCES}

1. Averey GB. Sepsis neonatorum. In: Gordon B. Averey, Mhairi G. MacDonald, Martha D. Mullet, eds. Text Book of Neonatology Pathophysiology \& Management of the Newborn. 6th ed. Philadelphia: Lippincott Williams and Wilkins; 2005: 1196-1207.

2. Sriram R. Correlation of blood culture results with the sepsis score and the sepsis screen in the diagnosis of neonatal septicaemia. Int $\mathbf{J}$ Biol Med Res. 2011;2(1):360-8.

3. Manjushree H. Bhalchandra, Harish Ghogare, Anupama Wyawahare, Vijay M. Mulay, Vishwesh P. Bansal, Mangala P. Bansal. Clinical and microbiological profile of neonatal sepsis at tertiary care hospital, Aurangabad, Maharastra. Natl J Med Sci. 2012;1(2):32-9.

4. Shah MN, Desai PB. Clinical and bacteriological profiles of blood culture positive sepsis in newborns. Int J Pharm Life Sci. 2011;2(9):1041-5.

5. Jiang JH, Chiu NC, Huang FY, Kao HA, Hsu CH, Hung HY, et al. Neonatal sepsis in the neonatal intensive care unit: characteristics of early versus late onset. J Microbiol Immunol Infect. 2004;37:301-6.

6. Ozkan H, Cetinkaya M, Koksal N, Celebi S, Hacimustafaoglu M. Culture-proven neonatal sepsis in preterm infants in a neonatal intensive care unit over a 7 year period, CoNS as a predominant pathogen. Paediatr Int. 2014;56:60-6.

7. Motara F, Ballot DE, Perovic O. Epidemiology of neonatal sepsis at Johennesburg Hospital. South Afr J Epidemiol Infect. 2005;20(3):90-3. 
8. Jiang JH, Chiu NC, Huang FY, Kao HA, Hsu CH, Hung HY, et al. Neonatal sepsis in the neonatal intensive care unit: characteristics of early versus late onset. J Microbiol Immunol Infect. 2004;37:301-6.

9. Gheibi S, Fakoor Z, Karamyyar M, Khashabi J, Ilkhanizadeh B, Asghari-Sana F, et al. Coagulase negative staphylococcus; the most common cause of neonatal septicemia in Urmia, Iran. Iran J Paediatr. 2008;18(3):237-43.

10. Katiyar R, Bose S. Bacteriological profile of neonatal septicaemia in Pravara Rural Hospital. Pravara Med Rev. 2012;4(2):4-6.
11. Mustafa M, Ahmed SL. Bacteriological profile and antibiotic susceptibility patterns in neonatal septicaemia in view of emerging drug resistance. J Med Allied Sci. 2014;4(1):2-8.

12. Mane AK, Nagdeo NV, Thombare VR. Study of neonatal septicemia in a tertiary care hospital in rural Nagpur. J Recent Adv Appl Sci. 2010;25:1924.

Cite this article as: Sarangi KK, Pattnaik D, Mishra SN, Nayak MK, Jena J. Bacteriological profile and antibiogram of blood culture isolates done by automated culture and sensitivity method in a neonatal intensive care unit in a tertiary care hospital in Odisha, India. Int J Adv Med 2015;2:387-92. 